\title{
ORIGINAL ARTICLE Metabolic phenotype-microRNA data fusion analysis of the systemic consequences of Roux-en-Y gastric bypass surgery
}

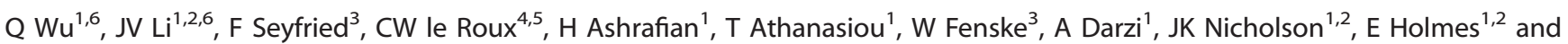 \\ NJ Gooderham ${ }^{1}$
}

BACKGROUND/OBJECTIVES: Bariatric surgery offers sustained marked weight loss and often remission of type 2 diabetes, yet the mechanisms of establishment of these health benefits are not clear.

SUBJECTS/METHODS: We mapped the coordinated systemic responses of gut hormones, the circulating miRNAome and the metabolome in a rat model of Roux-en-Y gastric bypass (RYGB) surgery.

RESULTS: The response of circulating microRNAs (miRNAs) to RYGB was striking and selective. Analysis of 14 significantly altered circulating miRNAs within a pathway context was suggestive of modulation of signaling pathways including $\mathrm{G}$ protein signaling, neurodegeneration, inflammation, and growth and apoptosis responses. Concomitant alterations in the metabolome indicated increased glucose transport, accelerated glycolysis and inhibited gluconeogenesis in the liver. Of particular significance, we show significantly decreased circulating miRNA-122 levels and a more modest decline in hepatic levels, following surgery. In mechanistic studies, manipulation of miRNA-122 levels in a cell model induced changes in the activity of key enzymes involved in hepatic energy metabolism, glucose transport, glycolysis, tricarboxylic acid cycle, pentose phosphate shunt, fatty-acid oxidation and gluconeogenesis, consistent with the findings of the in vivo surgery-mediated responses, indicating the powerful homeostatic activity of the miRNAs. CONCLUSIONS: The close association between energy metabolism, neuronal signaling and gut microbial metabolites derived from the circulating miRNA, plasma, urine and liver metabolite and gut hormone correlations further supports an enhanced gut-brain signaling, which we suggest is hormonally mediated by both traditional gut hormones and miRNAs. This transomic approach to map the crosstalk between the circulating miRNAome and metabolome offers opportunities to understand complex systems biology within a disease and interventional treatment setting.

International Journal of Obesity (2015) 39, 1126-1134; doi:10.1038/ijo.2015.33

\section{INTRODUCTION}

Obesity and its comorbidities have reached epidemic proportions across the developed and developing world, imposing an unsustainable socioeconomic burden on many societies. ${ }^{1}$ Bariatric surgery is the most effective treatment strategy for morbidly obese patients (body mass index $>40 \mathrm{~kg} \mathrm{~m}^{-2}$ ), or those with obesity comorbidities at a lower body mass index, as it can achieve sustained long-term weight loss and place type 2 diabetes in remission within days. ${ }^{2}$ Unlike diet-induced weight loss, Rouxen-Y gastric bypass (RYGB) achieves substantial weight loss with enhanced satiety, decreased hunger and increased energy expenditure while food restriction and malabsorption have not been identified as major players, in addition to reduced food intake. ${ }^{3}$ However, although the anatomical rearrangement leads to enterohormonal changes, altered bile flow ${ }^{4}$ profound changes of the gut microbiota ${ }^{5,6}$ and downregulation of endocannabinoids, the mechanism by which RYGB modulates metabolic pathways in an integrated way and the molecules responsible for coordinating these effects remain largely unknown.

MicroRNAs (miRNAs) are non-coding RNAs, 18-25 nucleotides in length, which regulate thousands of genes at the posttranscriptional level; ${ }^{8}$ each miRNA can potentially target multiple
mRNAs. This multi-targeting feature of miRNAs defines their unique role in governing multiple metabolic processes simultaneously. With their ability to influence pathway networks and high expression level in cells, miRNAs are believed to confer biological system robustness under homeostatic disturbance. ${ }^{9}$ We hypothesized that following RYGB surgery, which profoundly changes nutrients and bile flow, the individual establishes a new metabolic balance via the manipulation of miRNAs. Therefore, we profiled and integrated the plasma miRNAome and metabolome from Sprague Dawley (SD) rats undergoing RYGB surgery using a Statistical HeterospectroscopY (SHY) method, ${ }^{10}$ aiming to probe the composition of circulating miRNAs, which could behave as master metabolic regulators mediating post RYGB biological effects. Subsequent downstream in vitro model was used to probe these miRNA-mediated metabolic pathway alterations post RYGB surgery.

\section{MATERIALS AND METHODS}

Experiment design and sample collection

The animal experiment was carried out under a UK home office licence $\mathrm{PL}$ 70-6669). Thirteen male SD rats were individually housed and kept under a

${ }^{1}$ Division of Computational and Systems Medicine, Department of Surgery and Cancer Imperial College London, South Kensington, London, UK; ${ }^{2}$ Center for Digestive and Gut Health, Institute of Global Health Innovation, Imperial College London, London, UK; ${ }^{3}$ Department of General and Visceral, Vascular and Pediatric Surgery, University Hospital of Würzburg, Würzburg, Germany; ${ }^{4}$ Diabetes Complications Research Centre, Pathology, Conway Institute, School of Medicine and Medical Sciences, University College Dublin, Dublin, Ireland and ${ }^{5}$ Investigative Science, Imperial College London, London, UK. Correspondence: Professor E Holmes or Professor NJ Gooderham, Division of Computational and Systems Medicine, Department of Surgery and Cancer, Faculty of Medicine, Imperial College London, Sir Alexander Fleming Building, South Kensington, SW7 2AZ London, UK. E-mail: elaine.holmes@imperial.ac.uk or n.gooderham@imperial.ac.uk

${ }^{6}$ These authors contributed equally to this work.

Received 6 January 2015; accepted 22 February 2015; accepted article preview online 18 March 2015; advance online publication, 28 April 2015 
$12 \mathrm{~h} / 12 \mathrm{~h}$ light/dark cycle at room temperature. All rats were acclimatized and fed high-fat diet for 1 week prior to the experiment. Animals were randomly divided into two groups: RYGB $(n=8)$ and SHAM $(n=5)$. The antibiotic combination amoxicillin/flucoxacillin was administrated presurgery to all rats at a dose of $12.5 \mathrm{mg}$ per rat.

Animal body weight (BW) and food intake were measured daily. The gut hormones (for example, peptide YY (PYY) and glucagon-like peptide 1 (GLP-1)) were measured on the day of killing. Blood was collected from all groups in the fasting state via cardiac puncture under terminal anesthesia in tubes containing EDTA and dipeptidyl peptidase- 4 inhibitor. The plasma fraction was separated by centrifugation at $4{ }^{\circ} \mathrm{C}$ and stored at $-80^{\circ} \mathrm{C}$. All samples were assayed in duplicate. PYY-like inmmunoactivity was measured with a specific and sensitive radioimmunoassay, which measure both the full length (PYY1-36) and the fragment (PYY3-3^). GLP-1 was measured in duplicate by established in-house radioimmunoassay (Millipore, Billerica, MA, USA).

Spot urinary samples were collected at day 52 and all rats were culled 53 days after the surgery. Approximately $5 \mathrm{ml}$ of whole blood was taken from the heart, $2.5 \mathrm{ml}$ of which was transferred into an EDTA-containing tube (BD Bioscience, Oxford, UK) and the remaining to a sodium heparincoated tube. After a gentle shake, they were immediately centrifuged at $3500 \mathrm{~g}$ at room temperature for $10 \mathrm{~min}$. The resulting plasma samples were collected into two 1.5-ml RNase-free Eppendorf tubes, separately. The left lobe of the liver was collected from each rat. All samples were immediately snap-frozen in liquid nitrogen and stored at $-80^{\circ} \mathrm{C}$.

\section{Sample preparation for NMR spectroscopic analyses}

Plasma samples collected using sodium heparin and urine were thoroughly defrosted and vortexed for $15 \mathrm{~s}$. A total of $30 \mu \mathrm{l}$ of urine was mixed with $25 \mu \mathrm{l}$ of $0.2 \mathrm{M}$ sodium phosphate buffer in deuterium oxide $\left(0.01 \%\right.$ of sodium 3 -(trimethylsilyl) propionate-2,2,3,3- $\left.d_{4}, \mathrm{pH}=7.4\right)$, and $50 \mu \mathrm{l}$ of the mixture was transferred into a nuclear magnetic resonance (NMR) tube with an outer diameter of $1.7 \mathrm{~mm}$ for further spectroscopic analysis. A total of $400 \mu \mathrm{l}$ of plasma was mixed with $250 \mu \mathrm{l}$ of saline containing $20 \%$ deuterium oxide for the magnetic field lock. The resulting mixture was centrifuged at $10000 \mathrm{~g}$ for $10 \mathrm{~min}$, and $600 \mu \mathrm{l}$ of supernatant was transferred into a NMR tube with an outer diameter of $5 \mathrm{~mm}$ pending ${ }^{1} \mathrm{H}$ NMR spectral acquisition. The liver tissue extraction is described in $\mathrm{SI}$ Materials and Methods. The dry extracts of liver aqueous phase were resuspended in a mixture of $100 \mu \mathrm{l}$ water, $400 \mu \mathrm{l}$ deuterium oxide and $400 \mu \mathrm{l}$ aforementioned sodium phosphate buffer, centrifuged for $10 \mathrm{~min}$ at $10000 \mathrm{~g}$, and $600 \mu \mathrm{l}$ of supernatant was transferred into a NMR tube.

${ }^{1} \mathrm{H}$ Nuclear magnetic resonance spectroscopy of urine, plasma and liver extracts, and spectral data analysis

${ }^{1} \mathrm{H}$ NMR spectra of urine, plasma and liver aqueous extract samples were obtained using a Bruker $600 \mathrm{MHz}$ spectrometer (Bruker; Rheinstetten, Germany) at the operating ${ }^{1} \mathrm{H}$ frequency of $600.13 \mathrm{MHz}$ with a temperature of $300 \mathrm{~K}$. A standard NMR pulse sequence (recycle delay (RD) $-90^{\circ}-\mathrm{t}_{1}-90^{\circ}-\mathrm{t}_{\mathrm{m}}$ $-90^{\circ}$-acquisition) was applied to acquire one-dimensional ${ }^{1} \mathrm{H}$ NMR spectral data, where $t_{1}$ was set to $3 \mu \mathrm{s}$ and $t_{\mathrm{m}}$ (mixing time) was set to $100 \mathrm{~ms}$. Suppression of the water peak was achieved using selective irradiation during RD of $2 \mathrm{~s}$ and $t_{\mathrm{m}}$. A 90 degree pulse was adjusted to $\sim 10 \mu \mathrm{s}$. A total of 128 scans for plasma samples and 256 scans for liver aqueous extracts were collected into $64 \mathrm{k}$ data points with a spectral width of $20 \mathrm{ppm}$. A Carr-Purcell-Meiboom-Gill pulse sequence (RD- $90^{\circ}-\left(\tau-180^{\circ}-\tau\right)_{n}$-acquisition) was applied additionally to plasma samples to better visualize the signals of the low molecular weight metabolites. For the Carr-PurcellMeiboom-Gill experiment, a spin relaxation delay $(2 \mathrm{n \tau})$ of $64 \mathrm{~ms}$ was used. ${ }^{1} \mathrm{H}$ NMR spectral data processing and multivariate statistical analysis are described in Supplementary Information Materials and Methods.

\section{RNA isolation, reverse transcription and preamplification for}

Taqman miRNA low-density array

Total RNAs in plasma and the liver homogenates were extracted using a mirVANA PARIS RNA isolation kit (Ambion, Paisley, UK). The procedures for the liver RNA extraction followed the manufacturer's instruction, whereas for plasma samples, minor modifications were applied to better eliminate plasma protein. In brief, $100 \mu \mathrm{l}$ of the plasma sample was diluted with $100 \mu \mathrm{l}$ cell disruption buffer and then $200 \mu \mathrm{l}$ of $2 \times$ denature solution was added. The remaining extraction steps are the same as the manufacturer's instruction. RNAs from both plasma and liver tissues were eluted from
mirVANA PARIS columns using $100 \mu \mathrm{l}$ of $95^{\circ} \mathrm{C}$ DNase and RNase-free water and stored at $-20^{\circ} \mathrm{C}$ pending analysis.

Total RNA was reverse transcribed using Taqman Megaplex pools. Reverse transcription (RT) mix was prepared following the manufacturer's instructions. RT mix $(4.5 \mu \mathrm{l})$ was mixed with $3 \mu \mathrm{l}$ total RNA extracted from plasma. The RT products were preamplified according to the manufacturer's recommendations. RT product $(2.5 \mu \mathrm{l})$ was combined with $12.5 \mu \mathrm{l}$ Taqman PreAmp Master Mix $(2 \times)$ and $2.5 \mu \mathrm{l}$ Megaplex PreAmp Primers $(10 \times)$ to generate a final $25 \mu \mathrm{l}$ preamplification PCR mix. Preamplification was carried out following the manufacturer's instructions. Preamplification PCR product $(10 \mu \mathrm{l})$ was diluted with $30 \mu \mathrm{l} 0.1 \times$ TE buffer (pH 8.0). The diluted preamplification PCR product was stored at $-20^{\circ} \mathrm{C}$ until required.

\section{Taqman miRNA low-density array}

Taqman Array Rodent Card is able to detect all 373 miRNAs expressed in rat according to the Sanger miRBase v15. Each complete assay contain two sets of cards (Card A and B). Card A detects well-characterized miRNAs, which tend to be function defined and broadly/highly expressed. Card B detects most of the recently discovered, less functional defined and narrowly or low-expressed miRNAs. Diluted preamplification product $(9 \mu \mathrm{l})$ was combined with $450 \mu \mathrm{l}$ Taqman universal PCR Master Mix (no AmpErase UNG, $2 \times$ ) and $441 \mu$ l RNase/DNase-free water. Quantitative PCR reaction mix $(100 \mu l)$ was loaded into each port of the TaqMan MicroRNA Array. The array card was centrifuged at $331 \mathrm{~g}$ for $2 \mathrm{~min}$ and then mechanically sealed with Applied Biosystems 7900HT upgrade kit. Quantitative RT-PCR reaction was carried out using an Applied Biosystems 7900HT thermocycler according to the manufacturer's recommended cycling conditions.

The single Taqman miRNA assay, mRNA target prediction, protein extraction from the liver and Immunoblot, MiRDIAN miR-122 mimic transfection, and statistical correlation analysis among gut hormones, miRNAome and metabolome are described in SI Materials and Methods.

\section{RESULTS}

RYGB surgery alters gut hormone levels and metabolite profiles of urine, plasma and liver extracts in SD rats

RYGB surgery induced a reduction of $22.0 \%$ of pre-surgical BW in SD rats $(n=8)$ over 52 days (pre-surgery BW $=467.9 \pm 12.9$ g, postsurgery $B W=377.8 \pm 33.9 \mathrm{~g}$ ), whereas sham-operated animals $(n=5)$ had an average BW of $573.2 \mathrm{~g}$ over the experimental period (Supplementary Figure S1A). Consistent with previous studies, ${ }^{11}$ we observed that the plasma concentrations of gut hormones, GLP-1 and PYY, were significantly elevated following RYGB surgery $\left(\mathrm{GLP} 1_{\mathrm{RYGB}}=71.5 \pm 11.7 \mathrm{pmol}^{-1}, \mathrm{GLP}^{-1}{ }_{\mathrm{SHAM}}=10.5 \pm 3.3 \mathrm{pmol}^{-1}\right.$, $P<0.001 ; \mathrm{PYY}_{\mathrm{RYGB}}=26.2 \pm 5.6 \mathrm{pmoll}^{-1}, \mathrm{PYY}_{\mathrm{SHAM}}=10.4 \pm 4.8 \mathrm{pmoll}^{-1}$, $P<0.001$ ) (Supplementary Figures S1B and C).

Orthogonal-partial least squares-discriminant analysis (OPLS-DA) of ${ }^{1} \mathrm{H}$ nuclear magnetic resonance (NMR) spectroscopic data of urine, plasma and liver tissue collected 52 days post operation showed clear postoperative shift in metabolism in all matrices (Supplementary Figure S2, Table 1). Similar to our previous findings in Wistar rats and C57BL6 mice, urinary concentrations of citrate, fumarate, 2-oxoglutarate and succinate decreased post RYGB surgery, suggesting an enhancement in tricarboxylic acid cycle (TCA) activity. ${ }^{6,12}$ Increased urinary excretion of hostmicrobial co-metabolites including indoxyl sulfate, $p$-cresyl sulfate, $p$-cresyl glucuronide, phenylacetylglycine and trimethylamine $N$ oxide were observed. RYGB reduced plasma lipids and increased pyruvate, lactate and alanine, suggesting increased lipid metabolism and glycolysis. Elevations in the concentrations of hepatic glycogen, glucose, glycerol, lactate and alanine are consistent with the downregulation of gluconeogenesis and an increased conversion between glycogen and glucose. These observations of altered hormonal and metabolic status support extant literature and suggest that RYGB extensively regulates multiple energy metabolism-related pathways. 
Table 1. Plasma, urinary and hepatic aqueous metabolites found to be altered between RYGB- and SHAM-operated rats based on OPLS-DA models Plasma metabolite An OPLS-DA model of plasma CPMG spectral data $\left(Q^{2} Y=0.83 ; R^{2} X=33.5 \%\right)$

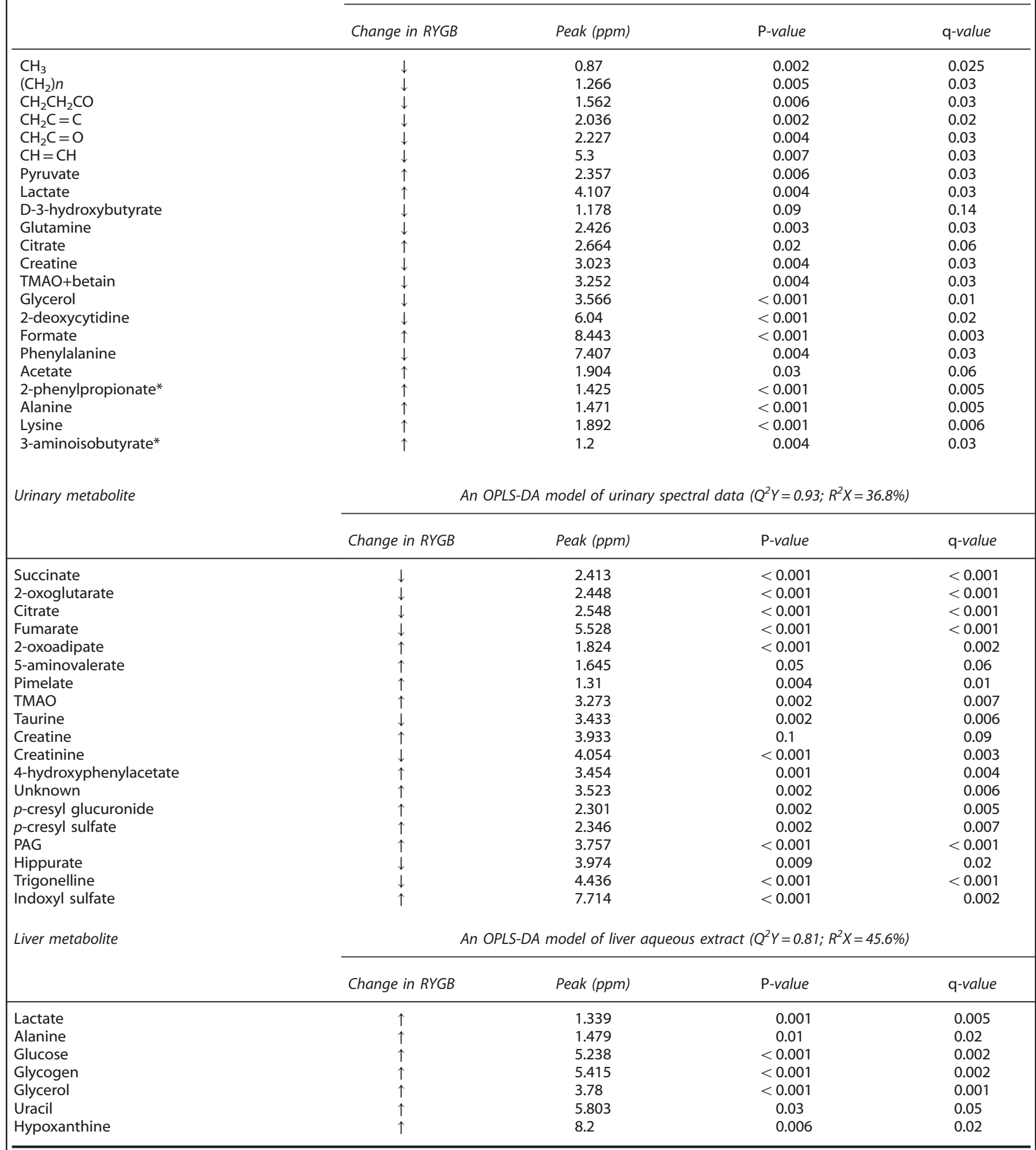

Abbreviations: CPMG, Carr-Purcell-Meiboom-Gill; OPLS-DA, orthogonal-partial least squares-discriminant analysis; PAG, phenylacetylglycine; RYGB, Roux-en-Y gastric bypass; TMAO, trimethylamine- $N$-oxide. $R^{2} X$ represents the variation in ${ }^{1} H$ NMR spectral data explained by the OPLS-DA model. $Q^{2} Y$ indicates the level of significance of the metabolic differences between two classes. The $P$ - and $q$-values in the table represent the significance of the metabolite changes and false discovery rate-adjusted $P$-values, respectively. The arrow up or arrow down represents the increased or decreased trend of metabolites in RYGB-operated rats compared with SHAM controls. *Tentative metabolite assignment. 
RYGB profoundly influences the global circulating miRNA expression in plasma

We next investigated the influence of RYGB on the circulating miRNAome using Taqman low-density array cards. Normalization is a key issue in circulating miRNA data analysis, thus we first developed a method for normalization of multiple-miRNAs and demonstrated its superiority over the widely used spike-in Caenorhabditis elegans cel-miR-39, global normalization and U6 normalization methods (as detailed in the Supplementary information, Supplementary Figure S3). Raw miRNA data were normalized using five endogenous miRNAs (U6-1, U6-2 miR-16, miR-223 and miR-1937b), and a total of 113 and 92 plasma circulating miRNAs were detected in four RYGB- and four SHAMoperated rats, respectively, 88 of which were common to both groups (Supplementary Table S1). Fourteen miRNAs were found to be significantly differentially expressed using a two-tailed student $t$-test $(P<0.05)$ (Figure $1 \mathrm{a})$. Eleven of the altered miRNAs were downregulated (miR-122, miR-93*, miR-872, miR-7*, miR-146a, miR-342-3p, miR-150, miR-139, miR-30a, miR-30e, miR-320), whereas three miRNAs, namely miR-463*, miR-34c* and miR-1188, were upregulated in the RYGB group. The clustered miRNA profiles show clear differentiation of the RYGB and SHAM groups (Figure 1b), wherein the RYGB-altered miRNAs typically exhibited a 1.5- to sixfold change compared with sham-operated rats, with the exception of miR-122, which demonstrated a 56 -fold $(P=0.0095)$ downregulation in RYGB-operated animals. This is consistent with a previous finding in RYGB patients, where miR-122 decreased significantly $(-94.2 \%, P<0.0001)$ when comparing baseline and after surgery miR-122 levels. ${ }^{13}$ Therefore, our data support the hypothesis that RYGB significantly alters circulating miRNAs, and thus the metabolic consequences of this require further investigation.

RYGB-altered plasma miRNAs influence multiple inflammation, obesity and neurodegenerative disease-related pathways

To gain insight into miRNA-mediated metabolic pathways affected by RYGB surgery, we predicted the mRNA targets of each of these altered circulating miRNAs with nine commonly used databases (See Materials and Methods) and retained only targets predicted by more than two programmes for further analysis. Based on the predicted pathways ranked according to the number of discriminatory miRNAs involved in each pathway (Figure 1c), we found that signaling pathways, specifically $G$ protein signaling, neurodegenerative, inflammation, and growth and apoptosis were highly likely to be influenced by the surgery. In particular, miR-342-3p, miR-320, miR-139-5p and miR-146a were predicted to be involved in multiple neurological transmitter and receptorrelated pathways, as well as two major neurodegenerative disease-associated pathways (Parkinson's and Huntingtons diseases), suggesting that RYGB surgery may modulate neurological activity through a miRNA-mediated gut-brain axis. Nine out of these 14 RYGB-altered miRNAs are associated with Parkinson's disease.

Correlation between miRNAome and the metabolic phenotype post RYGB surgery

Following the analysis of predicted miRNA-targeted pathways, we investigated the complex correlations between the circulating miRNAome and the plasma, urinary and hepatic metabolomes. We found that a number of distinctive sets of miRNAs from observed global circulating miRNAs were strongly correlated with plasma lipids, TCA cycle intermediates, host-gut microbial co-metabolites, liver and plasma energy metabolites (Figure 2) and gut hormones (Supplementary Figure S4). Downregulated plasma miR-342-3p and upregulated miR-34c* in RYGB-operated rats demonstrate a broad correlation with the liver, plasma and urinary metabolite profiles, indicating their extensive involvement in metabolic processes. Furthermore, miR-206, miR-1188, miR-1971 and miR$-34 c^{*}$ are inversely correlated with plasma lipid fractions, whereas miR-320 and miR-342-3p and to a lesser extent miR-7*, exhibit a positive correlation. TCA cycle intermediates including citrate, succinate, 2-oxoglutarate and fumarate are positively correlated with miR-143, miR-126-3p, miR-146a, miR-150 and miR-155. Interestingly, these same miRNAs are inversely correlated with urinary host-microbial co-metabolites such as $p$-cresyl glucuronide, $p$-cresyl sulfate and phenylacetylglycine. In addition to miR-342-3p and miR-34c*, miR-872* miR-463*, miR-30e, miR-2183, miR-1971, miR-150, miR-146a, miR-1188 and miR-93* all correlate with liver energy metabolism processes, such as glycolysis and glycogenesis involving glucose, glycogen and lactate.

Altered miR-122 expression levels in plasma and liver tissues are responsible for metabolic changes post RYGB surgery

RYGB induced the strongest effects on miR-122, as downregulation of miR-122 was observed in both bariatric patients ${ }^{13}$ and our rodent model for RYGB, we subsequently focused on investigating miR-122-mediated metabolic pathways. We first confirmed miR-122 expression levels in both plasma and liver tissue using Taqman real-time quantitative PCR (Figure 3a). The Pearson correlation value of the results derived from Taqman low-density array cards and quantitative PCR is $0.9983(P<0.0001)$. Plasma miR-122 showed a 99\% reduction in levels in RYGB-operated rats compared with SHAM controls, whereas hepatic miR-122 only showed $30 \%$ reduction of expression. We next examined a group of miR-122 targets, which are involved in key liver metabolic processes, such as the TCA cycle (Cs, citrate synthase), glucose transport (Glut1, glucose transporter protein type 1), pentose phosphate pathway (G6pd, glucose-6-phosphate dehydrogenase), fatty-acid synthesis (Fasn, fatty-acid synthase), energy sensing (Prkab1, adenosine monophosphate (AMP)-activated kinase beta 1), mitochondrial oxidation (Ucp2, uncoupling protein 2), gluconeogenesis (G6pc, glucose-6-phosphatase) and glycolysis (Aldoa, aldolase A). All mRNA targets are bona fide validated except for the putative targets UCP2 and AMPK 31 . These miR-122 targets in the liver all exhibited $>1.5$ fold increase in expression ranging from 1.53 to 1.91, except for Glut1 and G6pc. Although the alteration of Glut1 was not significant, it showed a clearly increased trend in the expression $(P=0.1061)$. G6pc expression, a validated miR-122 target, is surprisingly reduced $>80 \%$ in the RYGB group (Figure 3c). G6pc expression was lowered rather than elevated in the hepatic tissue, suggesting perhaps regulatory mechanisms of G6pc other than miR-122 predominate. We then investigated whether miR-122 can directly modulate these mRNA targets by manipulating their expression in the hepatocyte-like $\mathrm{B} 13 \mathrm{H}$ cell line (derived from a pancreatic AR42J-B13 cell line transdifferentiated with dexamethasone as primary hepatocytes are phenotypically unstable over the required time frame for the experiment). Transdifferentiation of this pancreatic cell to the $\mathrm{B} 13 \mathrm{H}$ cell derivative, results phenotypically in a liver-like cell expressing liver-specific and enriched functional enzymes. ${ }^{14}$ In fact, miR-122, believed to be a hepatocyte-specific miRNA, was upregulated more than 400 -fold during this trans-differentiation process (Supplementary Figure S5), consistent with the hepatic phenotype. In $\mathrm{B} 13 \mathrm{H}$ cells transfected with miR-122 mimic, expression of Cs, Glut1, Fasn, Aldoa, G6pd, Prkab1 and Ucp2 were all reduced (although the last three not significantly) in comparison with controls transfected with scrambled miRNA mimic (Figure $3 \mathrm{~d}$ ). Consistent with the aforementioned in vivo findings, the G6pc expression level was not affected by miR-122 in the $\mathrm{B} 13 \mathrm{H}$ cell line. We concluded that miR-122 directly modulated Cs, Glut1, Fasn, Aldoa and to a lesser extent, G6pd, Ucp2 and Prkab1. 
a

\begin{tabular}{cccc}
\hline microRNAs & Fold change & Change in RYGB & $p$ value \\
\hline miR-93\# & -3.3 & - & 0.0008 \\
miR-463\# & 5.7 & + & 0.0028 \\
miR-146a & -3.6 & - & 0.0029 \\
miR-122 & -56.8 & - & 0.0095 \\
miR-34c\# & 11.1 & + & 0.01 \\
miR-872\# & -1.8 & - & 0.0103 \\
miR-30a-3p & -1.8 & - & 0.0153 \\
miR-150 & -1.9 & - & 0.0162 \\
miR-7\# & -3.4 & - & 0.0164 \\
miR-139-5p & -2.1 & - & 0.0179 \\
miR-30e & -1.6 & - & 0.0219 \\
miR-320 & -3.3 & - & 0.0249 \\
miR-342-3p & -3.6 & - & 0.0374 \\
miR-1188 & 5.3 & + & 0.0377 \\
\hline
\end{tabular}

c

Neurodegenerative disease and neurological related

$G$ protein signalling related

Inflammation related

Growth and apoptosis related b

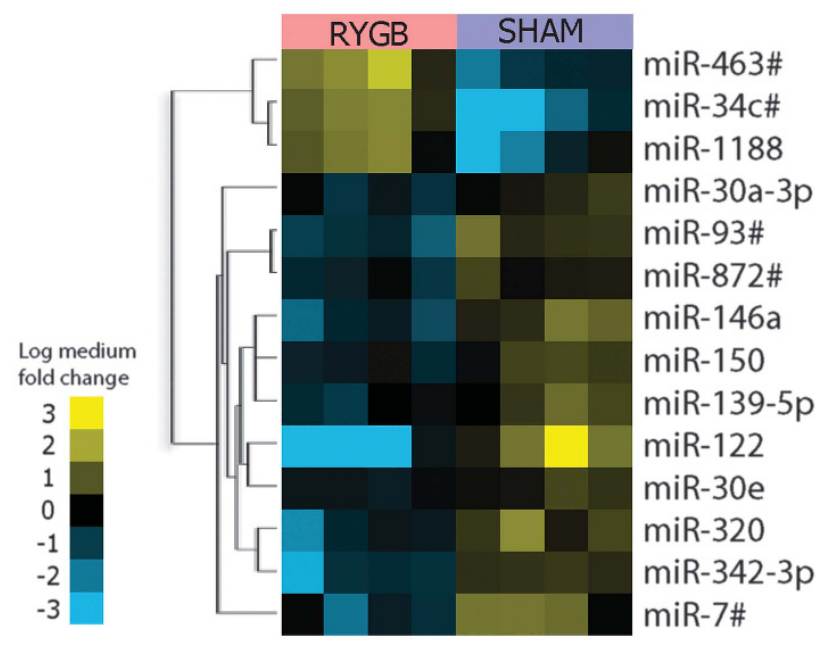

\section{$p<0.001$ \\ $0.001<p<0.01$ \\ $0.01<p<0.05$ \\ $p>0.05$}

Heterotrimeric G-protein signaling pathway-Gi alpha and Gs alpha mediated pathway
Parkinson disease
5 HT2 type receptor mediated signaling pathway
Huntington disease
Heterotrimeric G-protein signaling pathway-Gq alpha and Go alpha mediated pathway
Angiogenesis
PDGF signaling pathway
Synaptic_vesicle_trafficking
FGF signaling pathway
Inflammation mediated by chemokine and cytokine signaling pathway
Wnt signaling pathway
EGF receptor signaling pathway
T cell activation
VEGF signaling pathway
5 HT1 type receptor mediated signaling pathway
Histamine H1 receptor mediated signaling pathway
Metabotropic glutamate receptor group II pathway
Oxytocin receptor mediated signaling pathway
B cell activation
TGF-beta signaling pathway
Thyrotropin-releasing hormone receptor signaling pathway
PI3 kinase pathway
Apoptosis signaling pathway
Circadian clock system
Enkephalin release
Metabotropic glutamate receptor group III pathway
Interleukin signaling pathway

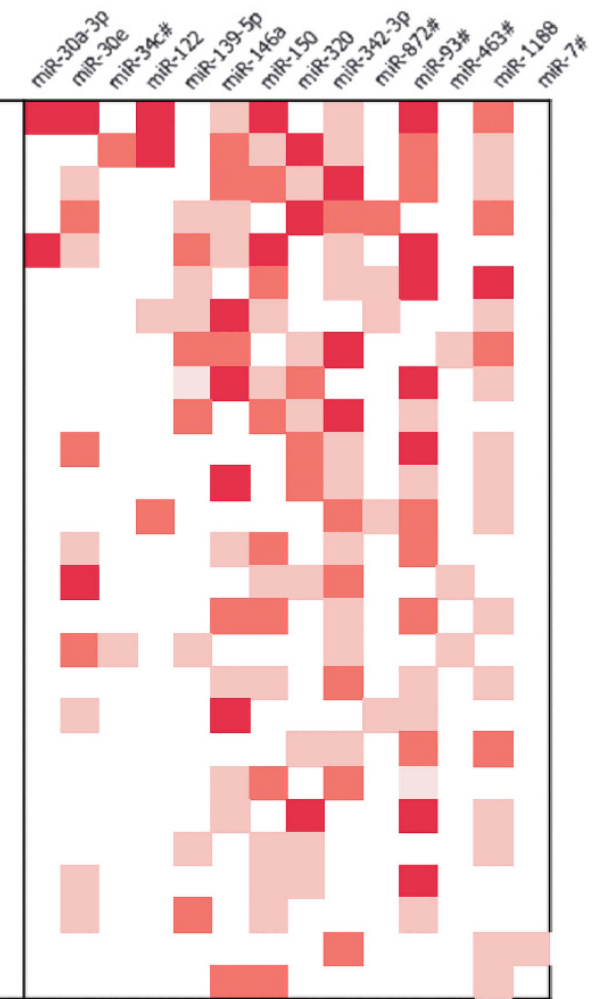

Figure 1. Altered circulating microRNAs and targeted signaling pathways. (a) The Roux-en-Y gastric bypass (RYGB) surgery altered the expression of 14 circulating microRNAs with 3 upregulated (yellow) and 11 downregulated (blue) in the RYGB-operated animals ( $n=4$ ) compared with sham controls $(n=4)$. $P$-values are derived from two-tailed $t$-test. (b) The heat map with two-way clustering shows clear grouping of RYGB and SHAM animals based on 14 significantly altered microRNAs. (c) The predicted pathways that these microRNAs can target are ranked according to the number of potentially involved microRNAs. P-values represent a statistical overrepresentation or underrepresentation of the genes in the predicted microRNA target list relative to the reference pathway gene list.

We confirmed these transcriptional effects on protein expression in the liver tissues of RYGB- and SHAM-operated rats (Figure 3f). CS, catalyzing the formation of citrate from oxaloacetate and acetyl coenzyme $A$, is a mitochondrial marker and has a key role in regulating energy generation in TCA cycle and mitochondrial respiration. We found that RYGB rats exhibited a twofold increase in CS protein expression (Figure 3g). UCP2 protein level showed a 2.6-fold upregulation in the RYGB animals (Figure 3h), consistent with the altered mRNA expression level (1.7-fold upregulation). AMPK acts as an energy switch; in an 

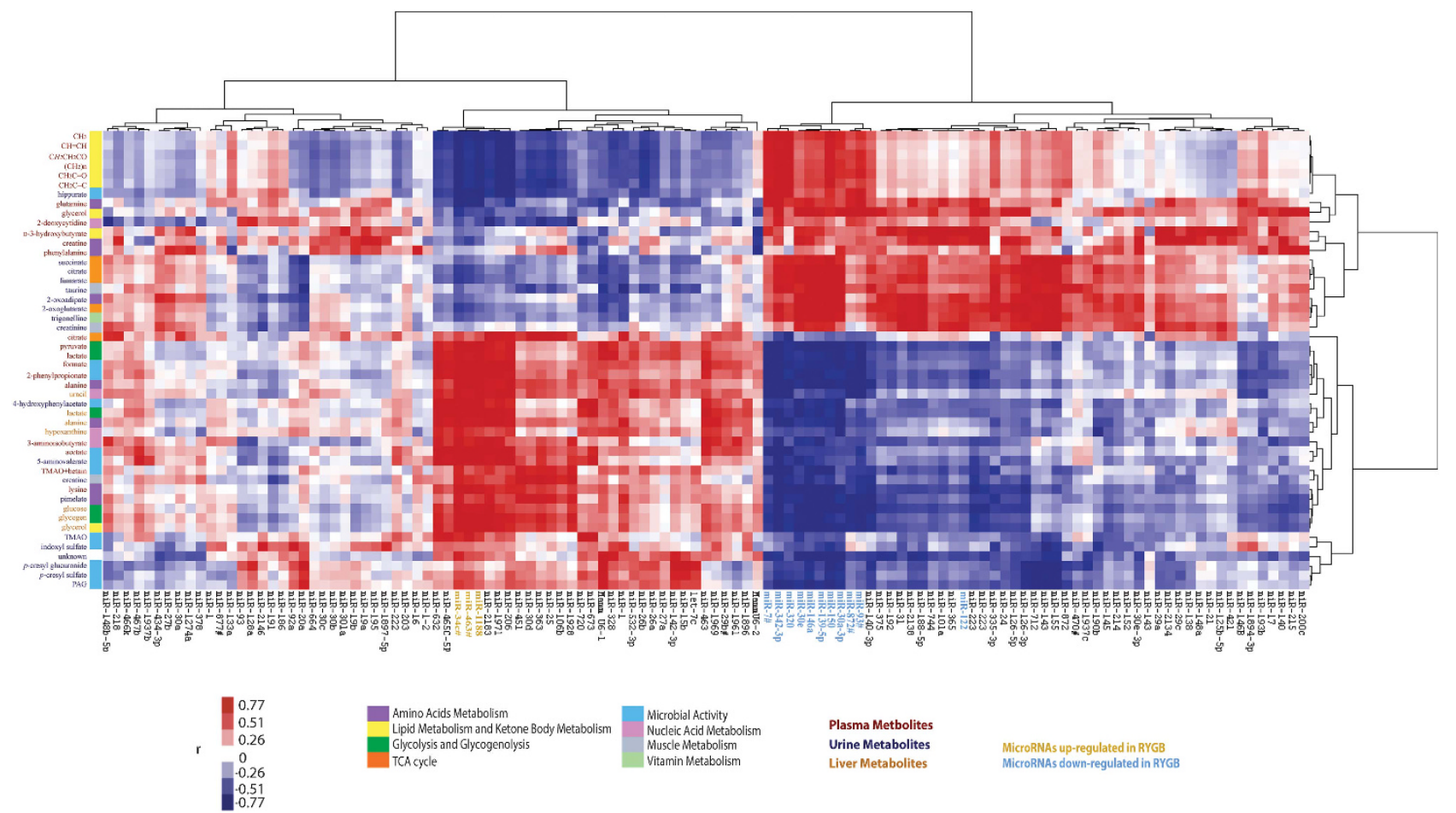

Figure 2. Correlation between miRNome and metabolic profiles. The heat map is generated from the Pearson correlation coefficient values between circulating microRNA expression and metabolite levels using a two-way clustering method. The horizontal axis shows all detectable circulating microRNAs, whereas the vertical axis exhibits altered plasma, urinary and hepatic metabolites following RYGB. The color bar next to the metabolites indicates metabolic functions (amino acid metabolism, lipid metabolism and ketone body metabolism, glycolysis and glycogenolysis, TCA cycles, microbial activity, nucleic acid metabolism, muscle metabolism and vitamin metabolism) of the metabolites. MicroRNAs, which are significantly altered by RYGB surgery, are shown in yellow (upregulated) and blue (downregulated), respectively. SHAM $n=4$, RYGB $n=4$. Keys: PAG, phenylacetylglycine; TMAO, trimethyalmine- $N$-oxide; TCA, tricarboxylic acid.

energy-deficient status (high AMP:ATP; adenosine triphosphate ratio), AMPK can switch off ATP-consuming biosynthesis pathways, such as Fasn in adipocytes, cholesterol synthesis and gluconeogenesis in the liver, and switch on ATP-producing catabolic pathways, for example, fatty-acid oxidation and glycolysis in multiple tissues. ${ }^{15}$ AMPK $\beta 1$ units require activation via phosphorylation. ${ }^{16}$ Although the non-phosphorylated-AMPK $\beta 1$ was downregulated by 2.5 fold, the active phosphorylatedAMPK $\beta 1$ protein was significantly upregulated by twofold (Figures $3 \mathrm{i}$ and $\mathrm{j}$ ).

\section{DISCUSSION}

Bariatric surgery has been shown to markedly alter circulating miRNAs in patients, in particular, downregulation of miR-122 by $94.7 \%{ }^{13}$ However, the metabolic functions and consequences of these dysregulated miRNAs have not been clarified or validated to date. We therefore investigated the correlation of the miRNAome and metabolites in a bariatric surgical model to elucidate energetic, microbial and neuronal signaling mechanisms of disease resolution. The mechanisms of RYGB surgery are complex; from our study and the work of others; it is apparent that circulating miRNAs could potentially work as a class of hormone, so-called miRormone, ${ }^{17}$ to mediate energy metabolism resulting in weight loss and potentially reset the brain-food rewarding system. Decreased plasma levels of miR-342-3p, miR-320, miR-139$5 p$ and miR-146a observed in our study suggest RYGB surgery impact on multiple neurodegenerative disease-related pathways. Patients with Parkinson's disease commonly experience unintended weight loss ${ }^{18}$ and factors including reduced food intake owing to dysphagia, increased resting energy expenditure and potential peptide hormones, such as ghrelin, could account for the underlying mechanisms. ${ }^{19}$ There is increased evidence suggesting a beneficial effect of bariatric operations on neurodegenerative disease based on surgical activity on caloric restriction, glycaemic control and gut hormonal modulation. ${ }^{20}$ The common miRNAtargeted pathways between bariatric surgery and Parkinson's disease suggest that surgery-induced weight loss may share certain mechanistic pathways with unintended weight loss in Parkinson's disease patients.

MiR-122 is a liver-produced miRNA and its less marked reduced expression in the liver compared with plasma suggests a restrained miR-122 release into the blood stream after RYGB surgery. By antagonizing miR-122 with LNA (locked-nucleic-acidmodified oligonucleotide)-anti-miR-122 in diet-induced obese mice for 6 weeks, a 30\% decrease in total cholesterol levels was achieved without lipid accumulation in the liver and tested miR-122 mRNA targets were similar to our findings except for $G 6 p c$, which was upregulated. ${ }^{21}$ Esau et al..$^{22}$ demonstrated that injecting miR-122 antisense oligonucleotide into mice for 5 weeks results in reduced plasma cholesterol levels, increased hepatic fatty-acid oxidation, decreased hepatic fatty-acid and cholesterol synthesis rates; effects that are also seen in primates. ${ }^{23}$ Following RYGB surgery, we observed increased glucose transportation, accelerated glycolysis and inhibited gluconeogenesis in the RYGB rat model. Glut1, a glucose transporter, was upregulated consistent with the decreased miR-122 expression in the liver and increased levels of hepatic glucose and glycogen were observed, indicating that RYGB promotes glucose transportation and glycogen synthesis. Our metabolic and miR-122 target expression data show that RYGB surgery suppresses gluconeogenesis and stimulates glycolysis, as evidenced by the 


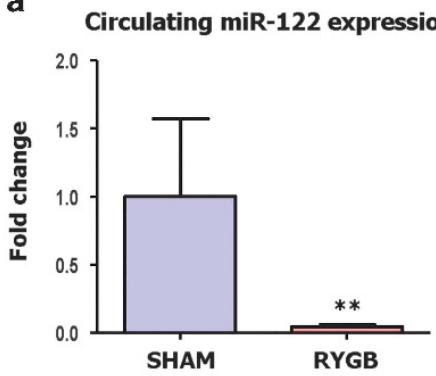

b

Hepatic miR-122 expression

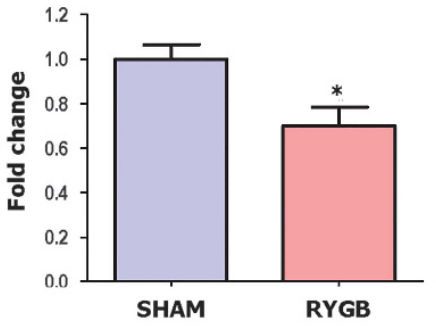

C

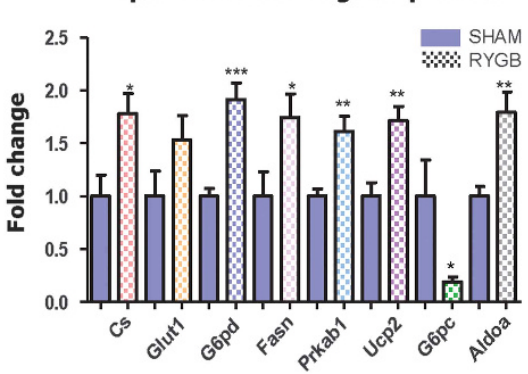

d

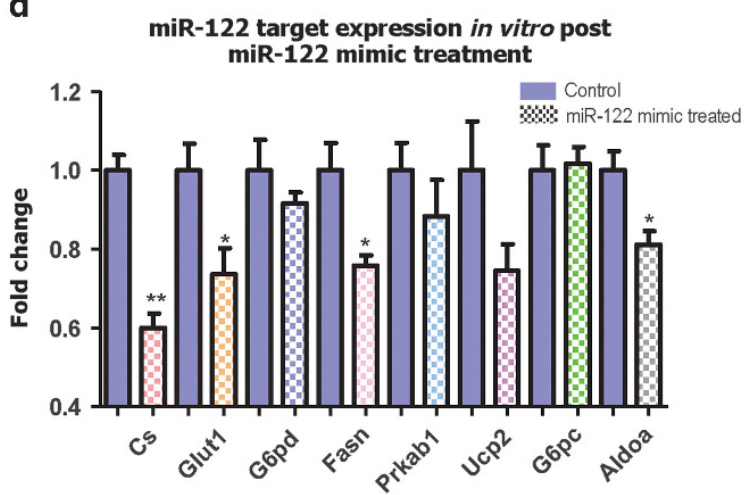

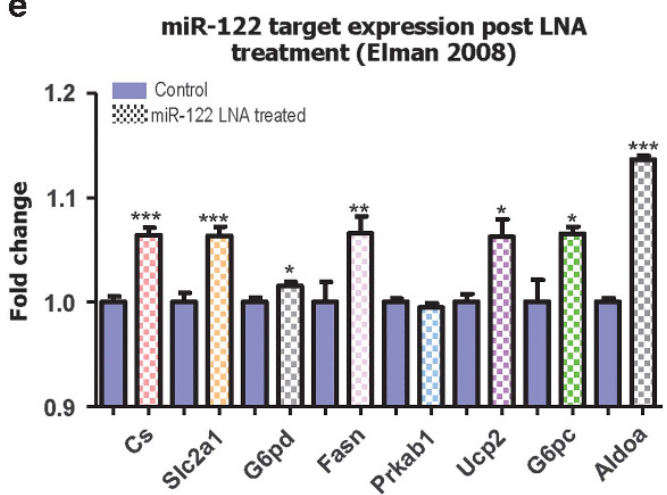

f
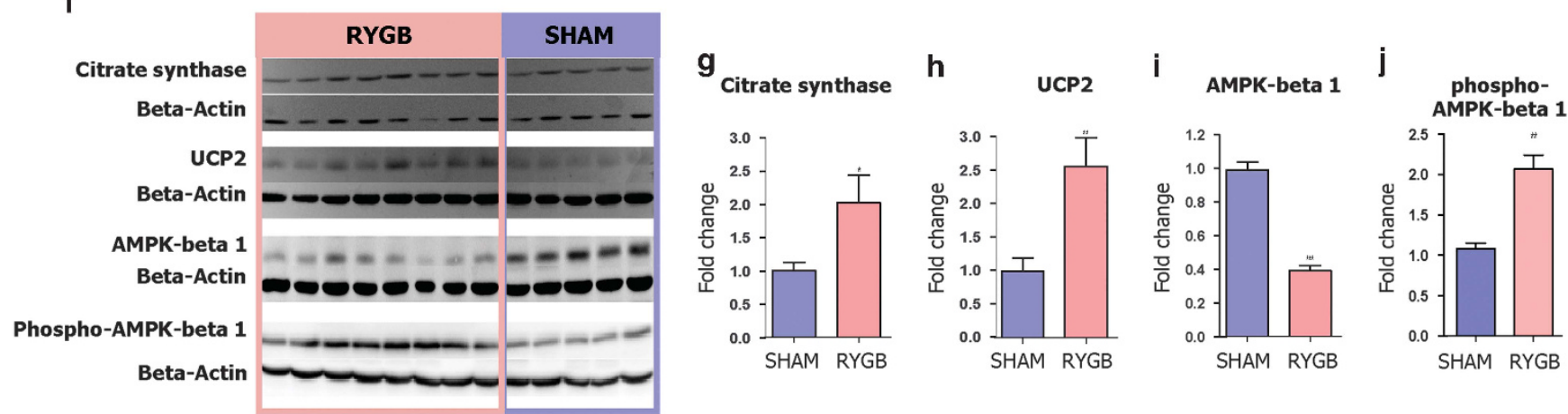

Figure 3. Expression levels of miR-122 and its targets in vitro and in vivo. The Roux-en-Y gastric bypass results in the altered miR-122 expression levels in plasma (a) and the liver (b) measured by quantitative PCR. (c) The expression levels of miR-122 mRNA targets, including citrate synthase (Cs), glucose transporter protein type 1 (Glut1), glucose-6-phosphate dehydrogenase (G6pd), fatty-acid synthase (Fasn), AMPactivated kinase beta 1 (Prkab1), uncoupling protein 2 (Ucp2), glucose-6-phosphatase (G6pc) and aldolase A (Aldoa), are measured by quantitative PCR in the liver of RYGB- $(n=8)$ and SHAM- $(n=5)$ operated animals. (d) These targets were evaluated in B13H cells transfected with miR-122 mimic (treatment, $n=4$ ) or scrambled miRNA (control, $n=4$ ) and measured by quantitative PCR. (e) A previous in vivo study by Elamn et al (Data were extracted from Array Express Experiment No. E-MEXP-1406, $n=5$ each group). (f) Immunoblot of the hepatic protein expression levels of citrate synthase, UCP2, AMPK-beta 1 and phosporylated AMPK-beta 1 in the RYGB- and sham-operated animals. $(\mathbf{g}-\mathbf{j})$ show quantified protein expressions of citrate synthase, UCP2, AMPK-beta 1 and phospho-AMPK-beta 1, respectively. All data represent mean \pm s.e.m. (RYGB, $n=8$; SHAM, $n=5$ ), unless otherwise specified in each figure. ${ }^{*} P<0.05 ;{ }^{* *} P<0.01 ;{ }^{* * *} P<0.001$.

downregulation of G6pc and upregulation of Aldoa, together with elevated concentrations of glycolysis end products including pyruvate, alanine and lactate in the liver and plasma (Figure 4). Mencarelli et al. ${ }^{24}$ have recently investigated the effects of ileal interposition, where the distal ileum is relocated into the proximal jejunum, which mimics a partial procedure of RYGB surgery. Consistent with our results, the hepatic expression of G6pc was also suppressed in this ileal interposition model. Furthermore, G6pd was found to be upregulated and this is an indicator for oxidative activity of the pentose phosphate pathway. It is likely that G6pd is stimulated by the unbalanced ratio of NADPH/NADP+ (normally 100:1 in the hepatic cytosol) to favor NADPH production. This unbalanced ratio could be because of the utilization of
$\mathrm{NADPH}$ in the liver by reductive biosynthesis, such as lipid biosynthesis. G6PD expression and enzyme activity have been reported to be upregulated in the Roux limb of RYGB-operated rats, as well as Glut $1,{ }^{25}$ and the authors concluded that the reprogramming of intestinal glucose, including enhanced basolateral glucose uptake, augmented glycolysis and stimulated pentose phosphate pathway, could contribute to the glycemic control after RYGB.

Decreased miR-122 levels induced the upregulation of CS (CS) and Ucp2 (UCP2). CS is a well-studied rate-limiting enzyme in the TCA cycle. Although the metabolic function of UCP2 is still controversial and varies between various types of tissues, it is believed to have a pivotal role in obesity, diabetes, metabolic 


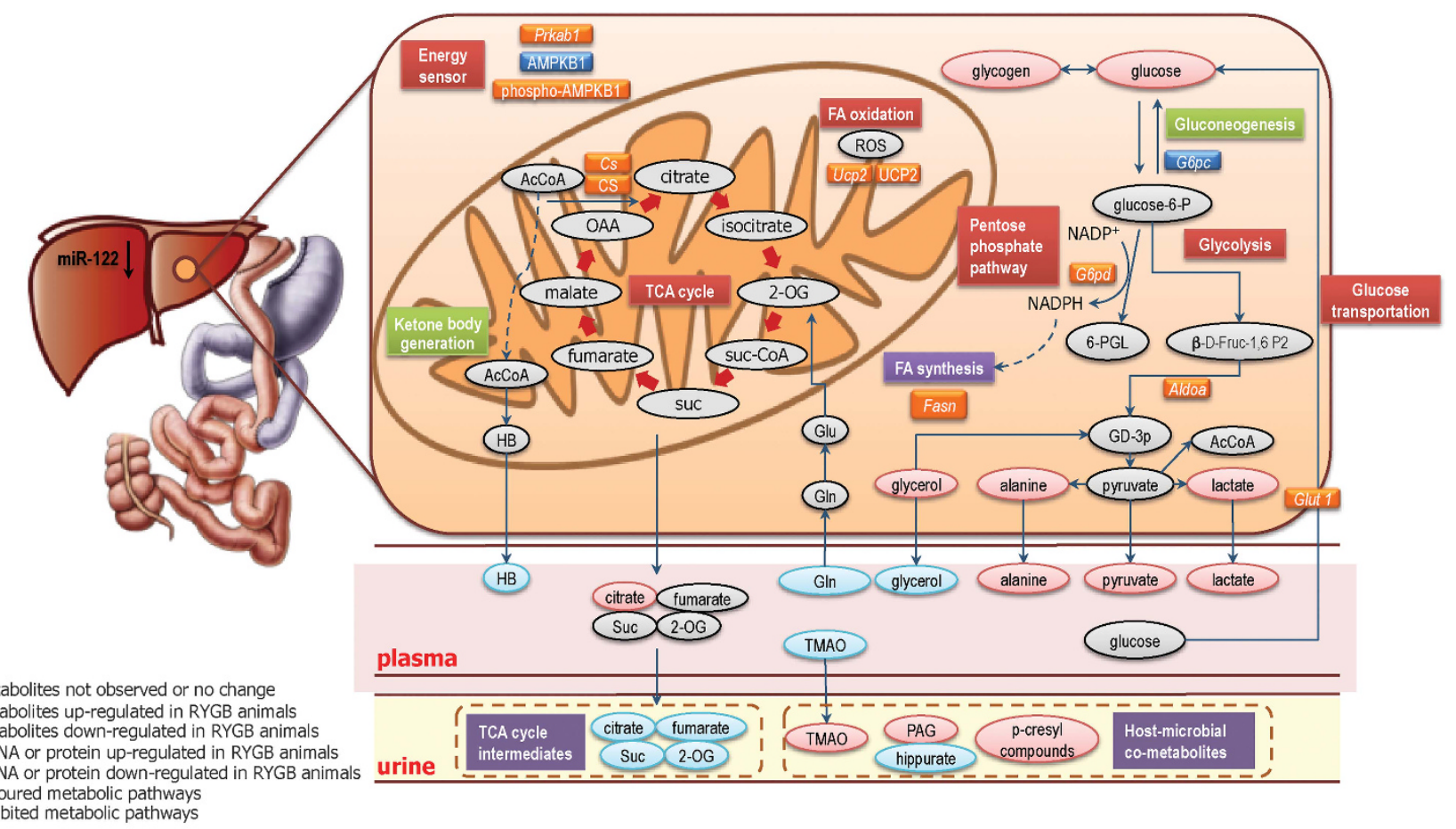

Figure 4. Modulation of metabolic activity by the downregulation of miR-122 following Roux-en-Y gastric bypass surgery. RYGB-induced metabolic changes in liver, plasma and urine (red and blue oval), metabolic enzymes (red and blue 3D boxes), and metabolic pathways (red and green flat boxes) are summarized. Keys: 2-OG, 2-oxoglutarate; 6-PGL, 6-phospho-D-glucono-1,5-lactone; AcCoA, Acetyl coenzyme A; Alodoa, aldolase A; AMP-activated kinase beta 1 (Prkab1, AMPK beta 1), $\beta$-D-Fruc-1,6 P2; Cs, citrate synthase; FA, fatty acids; Fasn, fatty-acid synthase; G6pc, glucose-6-phosphatase; G6pd, glucose-6-phosphate dehydrogenase; GD-3p, glyceraldehydes-3-phosphate; Gln, glutamine; Glu, glutamate; glucose-6-p, glucose-6-phosphatel; Glut1, glucose transporter protein type 1; HB, D-3-hydroxybutyrate; NADPH/NADP+, nicotinamide adenine dinucleotide phosphate; OAA, oxaloacetate; PAG, phenylacetylglycine; ROS, reactive oxygen species; suc, succinate; sucCoA, succinyl coenzyme A; TCA cycle, tricarboxylic acid cycle; TMAO, trimethylamine- $N$-oxide; Ucp2, uncoupling protein 2.

syndrome and several neurodegenerative diseases ${ }^{26,27}$ Hepatic UCP2, which uncouples oxidative phosphorylation with ATP production, is highly associated with oxidative phosphorylation and fatty-acid oxidation. UCP2 is responsible for proton transportation from the intermembrane space to the mitochondria matrix and uncouples proton flux with ATP synthesis. The upregulation of UCP2 and CS in the liver indicates that the RYGB-operated animals exhibit higher cellular metabolic activity such as favored fatty-acid oxidation and increased TCA cycle metabolism, which is further supported by the decreased levels of urinary TCA cycle intermediates detected by metabonomic analysis. Furthermore, UCP2 is a downstream effector of the energy sensor AMPK coded by the Prkab1 gene. In the present study, we also observed increased Prkab1 expression followed by upregulated UCP2. After RYGB, fat stored in adipose tissue is thought to be mobilized and metabolized in the liver, ${ }^{28}$ generating increased levels of free fatty acids, which could result in upregulated UCP2. It has been shown that increased UCP2 could not only promote the energy expenditure toward lipid utilization ${ }^{29}$ and compensate reduction of reactive oxygen species, $^{30}$ but also reduce oxidative stress ${ }^{31}$ and protect mitochondria during fatty-acid $\beta$-oxidation. ${ }^{32}$ This is consistent with decreased reactive oxygen species reported in patients after bariatric surgery. ${ }^{33,34}$ Hence, UCP2 and oxidative decoupling may contribute to postoperative weight loss. We observed that fatty-acid synthase (Fasn) also increased following RYGB surgery. The underlying reason for the increase in Fasn is unclear, but one possibility is that RYGB extensively affects lipid metabolism and leads to a restoration of adipose redox balance. Therefore, further studies should address lipid metabolism during the re-adjustment period after bariatric surgery.
In conclusion, we have demonstrated that RYGB surgery significantly modified metabolite and miRNA profiles of SD rats, resulting in substantial weight loss. We have demonstrated that miR-122 contributed to the control of energy metabolism with increased glucose transportation, glycolysis, TCA cycle, pentose phosphate shunt and fatty-acid oxidation and decreased gluconeogenesis and ketone body generation (Figure 4), suggesting an overall increased energy expenditure status. By correlating the circulating miRNAome and metabolome data, we were able to generate for the first time a comprehensive landscape of the crosstalk between miRNAs and metabolic pathways that point to a systemic regulation of processes including multiple axes of energy metabolism and suggested impact on the gut-brain axis. Followup studies will allow a detailed understanding of miRNAs responsible for regulating specific metabolic pathways, and conversely identifying metabolites capable of regulating the expression and activity of specific miRNAs. Our study highlights the value of transomic modeling and opens up a new window to further understand fundamental mechanisms associated with metabolic surgery and potential therapeutic targets for obesity and diabetes treatment.

\section{CONFLICT OF INTEREST}

The authors declare no conflict of interest.

\section{ACKNOWLEDGEMENTS}

Jia $\mathrm{V} \mathrm{Li}$ and the metabonomics study were supported by Imperial College Junior Research Fellowship. Qianxin Wu was supported by Imperial College International Student Scholarship and Kwok Foundation-Imperial College PhD Scholarship. Carel W le Roux supported by Science Foundation Ireland (12/Y1/B2480). 


\section{REFERENCES}

1 Yach D, Stuckler D, Brownell KD. Epidemiologic and economic consequences of the global epidemics of obesity and diabetes. Nat Med 2006; 12: 62-66.

2 Buchwald H, Avidor Y, Braunwald E, Jensen MD, Pories W, Fahrbach K et al. Bariatric surgery: a systematic review and meta-analysis. JAMA 2004; 292: 1724-1737.

3 Stylopoulos N, Hoppin AG, Kaplan LM. Roux-en-Y Gastric Bypass enhances energy expenditure and extends lifespan in diet-induced obese rats. Obesity 2009; 17: 1839-1847.

4 Ashrafian H, Bueter M, Ahmed K, Suliman A, Bloom SR, Darzi A et al. Metabolic surgery: an evolution through bariatric animal models. Obes Rev 2010; 11 907-920.

5 Zhang H, DiBaise JK, Zuccolo A, Kudrna D, Braidotti M, Yu Y et al. Human gut microbiota in obesity and after gastric bypass. PNAS 2009; 106: 2365-2370.

6 Li JV, Ashrafian H, Bueter M, Kinross J, Sands C, le Roux CW et al. Metabolic surgery profoundly influences gut microbial-host metabolic cross-talk. Gut 2011; 60: 1214-1223.

7 Guijarro A, Osei-Hyiaman D, Harvey-White J, Kunos G, Suzuki S, Nadtochiy S et al. Sustained weight loss after roux-en-y gastric bypass is characterized by down regulation of endocannabinoids and mitochondrial function. Ann Surg 2008; 247: 779-790.

$8 \mathrm{He} \mathrm{L}$, Hannon GJ. MicroRNAs: small RNAs with a big role in gene regulation. Nat Rev Genet 2004; 5: 522-531.

9 Ebert MS, Sharp PA. Roles for microRNAs in conferring robustness to biological processes. Cell 2012; 149: 515-524.

10 Crockford DJ, Holmes E, Lindon JC, Plumb RS, Zirah S, Bruce SJ et al. Statistical heterospectroscopy, an approach to the integrated analysis of NMR and UPLC-MS data sets: application in metabonomic toxicology studies. Anal Chem 2006; 78 363-371.

11 Roux CWL, Aylwin SJB, Batterham RL, Borg CM, Coyle F, Prasad V et al. Gut hormone profiles following bariatric surgery favor an anorectic state, facilitate weight loss, and improve metabolic parameters. Ann Surg 2006; 243: 108-114.

12 Seyfried F, Li JV, Miras AD, Cluny NL, Lannoo M, Fenske WK et al. Urinary phenotyping indicates weight loss-independent metabolic effects of Roux-en-Y gastric bypass in mice. J Proteome Res 2013; 12: 1245-1253.

13 Ortega FJ, Mercader JM, Catalán V, Moreno-Navarrete JM, Pueyo N, Sabater M et al. Targeting the circulating microRNA signature of obesity. Clin Chem 2013; 59 . 781-792.

14 Wallace K, Fairhall EA, Charlton KA, Wright MC. AR42J-B-13 cell: an expandable progenitor to generate an unlimited supply of functional hepatocytes. Toxicology 2010; 278: 277-287.

15 Hardie DG. AMPK: a key regulator of energy balance in the single cell and the whole organism. Int J Obes 2008; 32: S7-S12.

16 Mitchelhill KI, Michell BJ, House CM, Stapleton D, Dyck J, Gamble J et al. Posttranslational modifications of the 5'-AMP-activated protein kinase beta1 subunit. J Biol Chem 1997; 272: 24475-24479.

17 Cortez MA, Bueso-Ramos C, Ferdin J, Lopez-Berestein G, Sood AK, Calin GA. MicroRNAs in body fluids - the mix of hormones and biomarkers. Nat Rev Clin Oncol 2011; 8: 467-477.

18 Chen H, Zhang SM, Hernán MA, Willett WC, Ascherio A. Weight loss in Parkinson's disease. Ann Neurol 2003; 53: 676-679.

19 Pfeiffer RF. Gastrointestinal dysfunction in Parkinson's disease. Lancet Neurol 2003; 2: $107-116$.
20 Ashrafian H, Harling L, Darzi A, Athanasiou T. Neurodegenerative disease and obesity: what is the role of weight loss and bariatric interventions? Metab Brain Dis 2013; 28: 341-353.

21 Elmen J, Lindow M, Schutz S, Lawrence M, Petri A, Obad S et al. LNA-mediated microRNA silencing in non-human primates. Nature 2008; 452: 896-U10.

22 Esau C, Davis S, Murray SF, Yu XX, Pandey SK, Pear M et al. miR-122 regulation of lipid metabolism revealed by in vivo antisense targeting. Cell Metab 2006; 3: 87-98.

23 Elmén J, Lindow $M$, Silahtaroglu A, Bak M, Christensen $M$, Lind-Thomsen A et al Antagonism of microRNA-122 in mice by systemically administered LNA-antimiR leads to up-regulation of a large set of predicted target mRNAs in the liver. Nucleic Acids Res 2008; 36: 1153-1162.

24 Mencarelli A, Renga B, D'Amore C, Santorelli C, Graziosi L, Bruno A et al. Dissociation of intestinal and hepatic activities of FXR and LXRa supports metabolic effects of terminal ileum interposition in rodents. Diabetes 2013; 62 3384-3393.

25 Saeidi N, Meoli L, Nestoridi E, Gupta NK, Kvas S, Kucharczyk J et al. Reprogramming of intestinal glucose metabolism and glycemic control in rats after gastric bypass. Science 2013; 341: 406-410.

26 Zhang C-Y, Baffy G, Perret P, Krauss S, Peroni O, Grujic D et al. Uncoupling protein-2 negatively regulates insulin secretion and is a major link between obesity, $\beta$ cell dysfunction, and type 2 diabetes. Cell 2001; 105: 745-755.

27 Bechmann I, Diano S, Warden CH, Bartfai T, Nitsch R, Horvath TL. Brain mitochondrial uncoupling protein 2 (UCP2): a protective stress signal in neuronal injury. Biochem Pharmacol 2002; 64: 363-367.

28 Johansson L, Roos M, Kullberg J, Weis J, Ahlström H, Sundbom M et al. Lipid mobilization following Roux-en-Y gastric bypass examined by magnetic resonance imaging and spectroscopy. Obes Surg 2008; 18: 1297-1304.

29 Mattiasson G, Sullivan PG. The emerging functions of UCP2 in health, disease, and therapeutics. Antioxid Redox Signal 2006; 8: 1-38.

30 Castro MC, Massa ML, Del Zotto H, Gagliardino JJ, Francini F. Rat liver uncoupling protein 2: changes induced by a fructose-rich diet. Life Sci 2011; 89: 609-614.

31 Collins $\mathrm{P}$, Jones $\mathrm{C}$, Choudhury S, Damelin L, Hodgson H. Increased expression of uncoupling protein 2 in HepG2 cells attenuates oxidative damage and apoptosis. Liver Int 2005; 25: 880-887.

32 Patterson AD, Shah YM, Matsubara T, Krausz KW, Gonzalez FJ. Peroxisome proliferator-activated receptor alpha induction of uncoupling protein 2 protects against acetaminophen-induced liver toxicity. Hepatology 2012; 56: 281-290.

33 Ueda Y, Hajri T, Peng D, Marks-Shulman PA, Tamboli RA, Shukrallah B et al. Reduction of 8-iso-prostaglandin F2a in the first week after Roux-en-Y gastric bypass surgery. Obesity 2011; 19: 1663-1668.

34 Huang H, Kasumov T, Gatmaitan P, Heneghan HM, Kashyap SR, Schauer PR et al. Gastric bypass surgery reduces plasma ceramide subspecies and improves insulin sensitivity in severely obese patients. Obesity $2011 ; 19: 2235-2240$.

\section{(c) (i)}

This work is licensed under a Creative Commons Attribution 4.0 International License. The images or other third party material in this article are included in the article's Creative Commons license, unless indicated otherwise in the credit line; if the material is not included under the Creative Commons license, users will need to obtain permission from the license holder to reproduce the material. To view a copy of this license, visit http://creativecommons.org/licenses/ by/4.0/

Supplementary Information accompanies this paper on International Journal of Obesity website (http://www.nature.com/ijo) 